\title{
Clinical significance of microbial colonization identified by initial bronchoscopy in patients with lung cancer requiring chemotherapy
}

\author{
Ji Young Kang ${ }^{1}$, Hye Seon Kang ${ }^{1}$, Jung Won Heo ${ }^{1}$, Yong Hyun Kim ${ }^{1}$, Seung Joon Kim ${ }^{1,2}$, Sang Haak Lee ${ }^{1}$, \\ Soon Seog Kwon ${ }^{1}$, Youn Jeong $\mathrm{Kim}^{3} \wedge$ \\ ${ }^{1}$ Division of Pulmonary, Critical Care and Allergy, Department of Internal Medicine, College of Medicine, The Catholic University of Korea, Seoul, \\ Republic of Korea; ${ }^{2}$ Postech-Catholic Biomedical Engineering Institute, College of Medicine, The Catholic University of Korea, Seoul, Korea; \\ ${ }^{3}$ Division of Infectious Disease, Department of Internal Medicine, College of Medicine, The Catholic University of Korea, Seoul, Republic of Korea \\ Contributions: (I) Conception and design: JY Kang, YJ Kim; (II) Administrative support: JY Kang, HS Kang; (III) Provision of study materials \\ or patients: YH Kim, SJ Kim, SH Lee, SS kwon; (IV) Collection and assembly of data: JY Kang, HS Kang, JW Heo; (V) Data analysis and \\ interpretation: JY Kang, HS Kang, YJ Kim; (VI) Manuscript writing: All authors; (VII) Final approval of manuscripts. \\ Correspondence to: Youn Jeong Kim, MD, PhD. Department of Internal Medicine, Incheon St. Mary's Hospital, College of Medicine, The Catholic \\ University of Korea, 56, Dongsu-ro, Bupyeong-gu, Incheon 21431, Republic of Korea. Email: muze1004@catholic.ac.kr.
}

Background: There are limited data on the association between bronchial colonization and respiratory infections in people with lung cancer requiring cytotoxic chemotherapy. We investigated whether bronchial colonization in initial bronchoscopy specimens can predict the development of pneumonia after chemotherapy in patients with lung cancer.

Methods: Four hundred thirteen patients with lung cancer included in the Catholic Medical Center lung cancer registry were enrolled from March 2015 to August 2018. Demographic data, microbiology results, development of pneumonia after chemotherapy, and clinical information about lung cancer were analyzed retrospectively.

Results: A total of 206 lung cancer patients treated with chemotherapy were included in the analysis. Forty patients (19.4\%) had positive results for the bronchial washing culture during the initial evaluation of lung cancer. The most common organisms were Klebsiella pneumoniae $(\mathrm{n}=14)$ and Streptococcus pneumoniae $(\mathrm{n}=6)$ in the surveillance culture, and Pneumocystis jirovecii $(\mathrm{n}=12)$ and Staphylococcus aureus $(\mathrm{n}=8)$ at the time of pneumonia development. Eighty-nine patients (43.2\%) had pneumonia after chemotherapy, but the occurrence of pneumonia did not differ according to the colonization. There were no patients for whom the initial isolated organism was a causative microbe for the development of pneumonia after or during chemotherapy. The pneumonia group had poorer prognosis than the non-pneumonia group (378 vs. 705 days, $\mathrm{P}=0.0004)$.

Conclusions: Microbial colonization in bronchoscopy specimens was not associated with pneumonia development or mortality after chemotherapy for lung cancer. This finding suggests that testing surveillance culture may not be helpful for predicting pneumonia or improving survival in lung cancer patients with chemotherapy.

Keywords: Chemotherapy; colonization; lung cancer; pneumonia

Submitted Aug 22, 2020. Accepted for publication Dec 29, 2020.

doi: $10.21037 /$ jtd-20-2722

View this article at: http://dx.doi.org/10.21037/jtd-20-2722

^ ORCID: 0000-0001-5870-1801. 


\section{Introduction}

Pneumonia occurs often in cancer patients. For example, about $20 \%$ of leukemia patients receiving chemotherapy and $80 \%$ of hematopoietic stem cell transplant recipients experience pulmonary complications $(1,2)$. Lung cancer patients treated with cytotoxic chemotherapy are vulnerable to infection because of impaired immunity. Advanced age, poor performance status, the pathologic type of lung cancer (e.g., squamous cell carcinoma), obstructed pattern of lung function, and accompanied neutropenia are known risk factors for pneumonia during cytotoxic chemotherapy in lung cancer patients (3-5).

The use of surveillance culture is an effective strategy for infection control. Monitoring the presence of vancomycinresistant enterococci in a rectal swab or methicillin-resistant Stapbylococcus aureus (S. aureus) in a nasal swab is a widely used surveillance method because colonization of these microorganisms acts as a reservoir for transmission to other patients and is associated with mortality (1). In our previous study of liver transplant recipients, positive sputum culture results at the time of and 5 and 10 days after transplantation were associated with post-transplantation pneumonia (6). Mechanically ventilated patients with heavy tracheal colonization with $S$. aureus or gram-negative organims have a higher risk of developing ventilator-associated pneumonia by these microbes $(7,8)$. In two reports of patients with resectable lung cancer, preoperative bronchial colonization predicted postoperative pneumonia and correlated with mortality $(9,10)$. In real practice, when a patient with lung cancer develops pneumonia, it is not unusual for clinicians to choose specific antibiotics considering previous microbiologic results obtained from respiratory specimens.

There are limited data on the clinical efficacy of checking for bronchial colonization in lung cancer patients treated with chemotherapy $(11,12)$. The aim of this study was to investigate whether bronchial colonization observed by bronchoscopy during an initial examination would predict the development of pneumonia after chemotherapy in patients with lung cancer. We present the following article in accordance with the STROBE reporting checklist (available at http://dx.doi.org/10.21037/jtd-20-2722).

\section{Methods}

\section{Data source}

The Catholic Medical Center lung cancer registry is a web-based database in which all patients are enrolled when diagnosed with lung cancer in any of seven multicenters (Seoul St. Mary's Hospital, Yeouido St. Mary's Hospital, St. Paul's Hospital, Uijeongbu St. Mary's Hospital, Bucheon St. Mary's Hospital, Incheon St. Mary's Hospital, and St. Vincent's Hospital) in the South Korean capital region since October 2014. To improve the accuracy of the data, qualified data managers systematically record all clinical information including stage, pathology, treatment modality, and survival in this database. Using this registry, we reviewed the medical charts of patients diagnosed with lung cancer in Seoul St. Mary's Hospital and Bucheon St. Mary's Hospital from March 2015 to August 2018. This study was approved by the Clinical Research Ethics Committee of the Catholic Medical Center (XC140IMI0070).

\section{Data collection}

All patients who received a bronchoscopy examination and who had the microbiology results from bronchial specimens obtained during the initial evaluation of their lung cancer in the registry were enrolled retrospectively. Patients with concurrent pneumonia at the time of diagnosis of lung cancer were excluded. We collected baseline demographic characteristics including age, sex, body mass index (BMI), smoking history, comorbidities, pathologic type of lung cancer, presence of neutropenia, pulmonary function test results, clinical staging, treatment modalities, and line of therapy. We were especially interested in identifying patients who developed pneumonia after treatment with chemotherapy for their malignancy according to the initial bronchial colonization.

Respiratory samples were obtained by bronchial washing or brushing of the distal bronchi near the tumor lesion. To acquire a $20 \mathrm{ml}$ sample, $40-50 \mathrm{ml}$ of saline washing fluid was used. Routine microbiology evaluation included bacterial culture, polymerase chain reaction (PCR) tests for Mycoplasma pneumoniae and Mycobacterium tuberculosis, acid-fast bacilli stain/culture, and fungus culture. Samples from some patients were also analyzed using PCR methods to detect respiratory virus or Pneumocystis jirovecii ( $P$. jirovecii) according to the attending clinician's decision. For samples from patients who developed pneumonia after chemotherapy, similar microbial analyses were performed using bronchial samples or sputum if available. All microbiologic samples were processed for bacterial culture using a VITEK GNI automated system (bioMérieux, MO, USA). Species identification and antimicrobial susceptibility were tested using the VITEK GNI automated system 


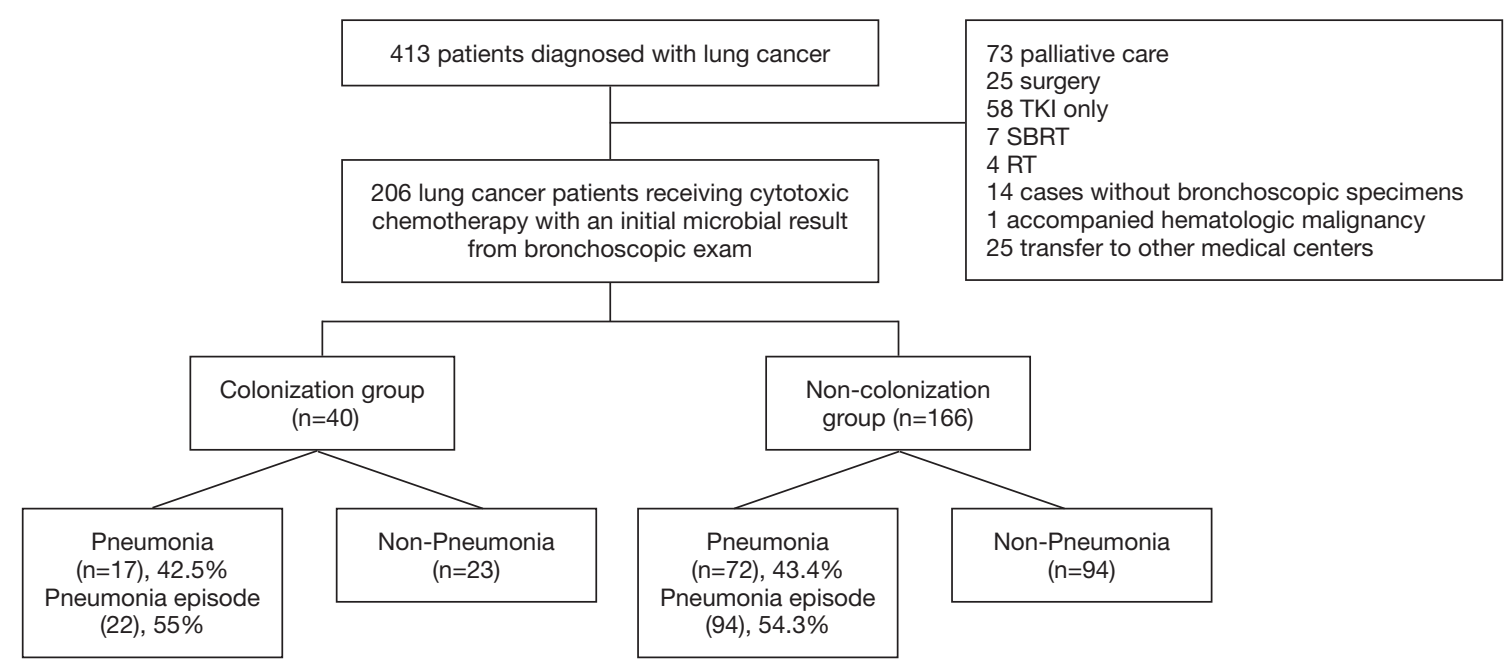

Figure 1 Study profile. TKI, tyrosine kinase inhibitor; SBRT, stereotactic body radiation therapy; RT, radiation therapy

according to Clinical and Laboratory Standards Institute criteria (13). Colonization was defined as isolation of microorganisms without the presence of typical pneumonia as reported in previous studies $(14,15)$.

\section{Criteria for diagnosing pulmonary infection}

Pneumonia was defined as the presence of new or progressive infiltration on chest radiography or computed tomography (CT), together with either unexplained fever or purulent sputum (1). Microbiologic evidence, including positive blood cultures or isolation of pathogen(s) from the sputum or bronchial specimens, was not a compulsory diagnostic criterion. The differential diagnosis of pulmonary infiltration included noninfectious mimics such as leukemic infiltration, drug toxicity, pulmonary edema or hemorrhage, or the progression of underlying malignancy. We also excluded radiation pneumonitis as shown on chest $\mathrm{CT}$ as a relatively sharp demarcated consolidation or ground-glass opacity within limited radiation fields.

\section{Statistical analysis}

Pearson's chi-square test or Fisher's exact test was used where appropriate to compare discrete variables, and Student's $t$ test or Mann-Whitney $U$ test was used to compare continuous variables. Survival analysis was performed using Kaplan-Meier analysis. A two-sided P value $<0.05$ was considered to be significant. The GraphPad Prism statistical software package (San Diego, CA, USA) was used for the analyses.

\section{Ethical statement}

The study was conducted in accordance with the Declaration of Helsinki (as revised in 2013). This study was approved by the Clinical Research Ethics Committee of the Catholic Medical Center (XC140IMI0070). Due to retrospective nature of this study, the need for patient consents was waived.

\section{Results}

A total of 413 patients were diagnosed with lung cancer. We excluded 73 of these patients who were under palliative care, 25 who underwent surgery, 58 who were treated with a tyrosine kinase inhibitor (TKI), seven who underwent stereotactic body radiation therapy, four who underwent palliative radiation therapy, 14 who did not have bronchoscopy evaluation for microbiology, one who had an accompanying hematologic malignancy, and 25 who were transferred to other medical centers. Finally, a total of 206 lung cancer patients who underwent a bronchoscopy examination at the initial evaluation of malignancy and were treated with cytotoxic chemotherapy were included in the analysis (Figure 1).

Among the 206 lung cancer patients, 120 patients underwent bronchial washing and 86 patients underwent bronchial washing and brushing. Forty patients (19.4\%) had positive microbial results in their bronchial specimens 
(microbial colonized group: 24 bronchial washing, 16 bronchial washing/brushing). The microbial colonized and noncolonized groups did not differ significantly on mean age, ratio of male to female patients, comorbidities, BMI, smoking history, type or staging of lung cancer, and pulmonary function such as forced expiratory volume in $1 \mathrm{~s}$ (Table 1). The most commonly isolated organisms were Klebsiella pneumoniae (K. pneumoniae) $(\mathrm{n}=14)$, followed by Streptococcus pneumoniae (S. pneumoniae) $(\mathrm{n}=6)$, S. aureus $(\mathrm{n}=5)$, nontuberculous mycobacteria (NTM) $(\mathrm{n}=4)$, Aspergillus species $(\mathrm{n}=3)$, Pseudomonas aeruginosa $(\mathrm{n}=2)$, and Candida glabrata $(\mathrm{n}=2)$ (Table 2).

A total of 89 patients $(43.2 \%)$ had 116 pneumonia episodes: 22 cases in the microbial colonized group $v s$. 94 cases in the noncolonized group. However, the rate of occurrence of pneumonia did not differ significantly between the two groups: $42.5 \%$ (17/40) vs. 43.4\% (72/166), respectively $(\mathrm{P}=0.920)$. The interval to the first development of pneumonia from the initial bronchoscopy for surveillance culture or from the initial chemotherapy date was shorter in the microbial colonized group than in the noncolonized group (median 129 vs. 174 days and 112 vs. 154 days, respectively), but the difference between groups was not significant (Table 3). The percentage of patients with severe pneumonia requiring admission were similar between the colonized and noncolonized groups: $37.5 \%(15 / 40) v s$. $39.8 \%(66 / 166)(\mathrm{P}=0.645)$.

In the microbial colonized group, 20 of 22 episodes of pneumonia after systemic chemotherapy had results data from a sputum study for the evaluation of the pathogens. Ten patients had positive microbial results: three exhibited P. jirovecii, three $S$. aureus, and one each M. pneumoniae, Acinetobacter baumannii, K. pneumoniae, Rotbia mucilaginosa, NTM, Aspergillus, cytomegalovirus, and influenza. Interestingly, in no case was the organism found in the initial culture the same as that isolated during the development of pneumonia. In five patients for whom Mycobacterium species were isolated using the culture method at the initial bronchoscopic examination, one patient with $M$. tuberculosis was treated with antituberculous medication and four patients with NTM (one with $M$. intracellulare, one with $M$. avium, and two with subspecies not identified) were not treated. During the follow-up, only one patient exhibited progression of NTM lung disease. In the evaluation of causative microbe in the patients with pneumonia, the most frequently cultured pathogens were, in order, P. jirovecii [12], S. aureus [8], NTM [6], and K. pneumoniae [5] (Table 4).
The overall mortality rate did not differ significantly between the colonized and noncolonized groups: $55.0 \%$ $(22 / 40)$ vs. $60.2 \%(100 / 166)$, respectively $(\mathrm{P}=0.545)$. Moreover, the pneumonia-related mortality rate did not differ significantly between the two groups: $15.0 \%(6 / 40)$ vs. $21.1 \%(35 / 166)$, respectively $(\mathrm{P}=0.387)$ (Table 3). Experiencing pneumonia during chemotherapy was associated with a shorter median overall survival: 378.0 days in the pneumonia group $v s .705$ days in the nonpneumonia group $(\mathrm{P}=0.0004)$ (Figure 2). The median follow-up time for the time of the onset of pneumonia and of overall survival were 636 days (95\% confidence interval, 532-739) and 1,054 days (868-1,241 days), respectively. The overall survival of patients with lung cancer requiring chemotherapy did not differ significantly according to the presence of microbial colonization in the initial bronchoscopy examination or occurrence of neutropenia during chemotherapy $(\mathrm{P}=0.676$ and $\mathrm{P}=0.361$, respectively; data not shown).

\section{Discussion}

This study investigated the clinical impact of bacterial colonization identified by the initial diagnostic bronchoscopy on later pneumonia development and clinical outcomes in lung cancer patients treated with cytotoxic chemotherapy. A positive microbial culture in the bronchial specimens was not associated with pneumonia occurrence or infection-related mortality during or after cytotoxic chemotherapy in these patients with lung cancer. Understandably, pneumonia occurrence was associated with poorer survival in the patients.

In the current study, the clinical impact of the initial bronchial colonization on the pneumonia incidence or overall survival was not significant in the patients with lung cancer treated with chemotherapy. Data on the association between bronchial colonization and respiratory infections in lung cancer are scarce, especially in those treated with systemic chemotherapy, as in our patients. In previous studies focusing on lung cancer patients undergoing major lung resection, bronchial colonization was associated with postoperative pneumonia, the need for mechanical ventilation, and longer stays in the intensive care unit and hospital $(10,16)$. In a prospective study that enrolled patients with lung cancer requiring treatment, colonized patients with lung cancer had a higher bronchopulmonary infection rate and poorer survival than noncolonized patients (12). By contrast, other studies reported that airway colonization is not related to pneumonia development, consistent 
Table 1 Demographic characteristics of lung cancer patients treated with systemic chemotherapy

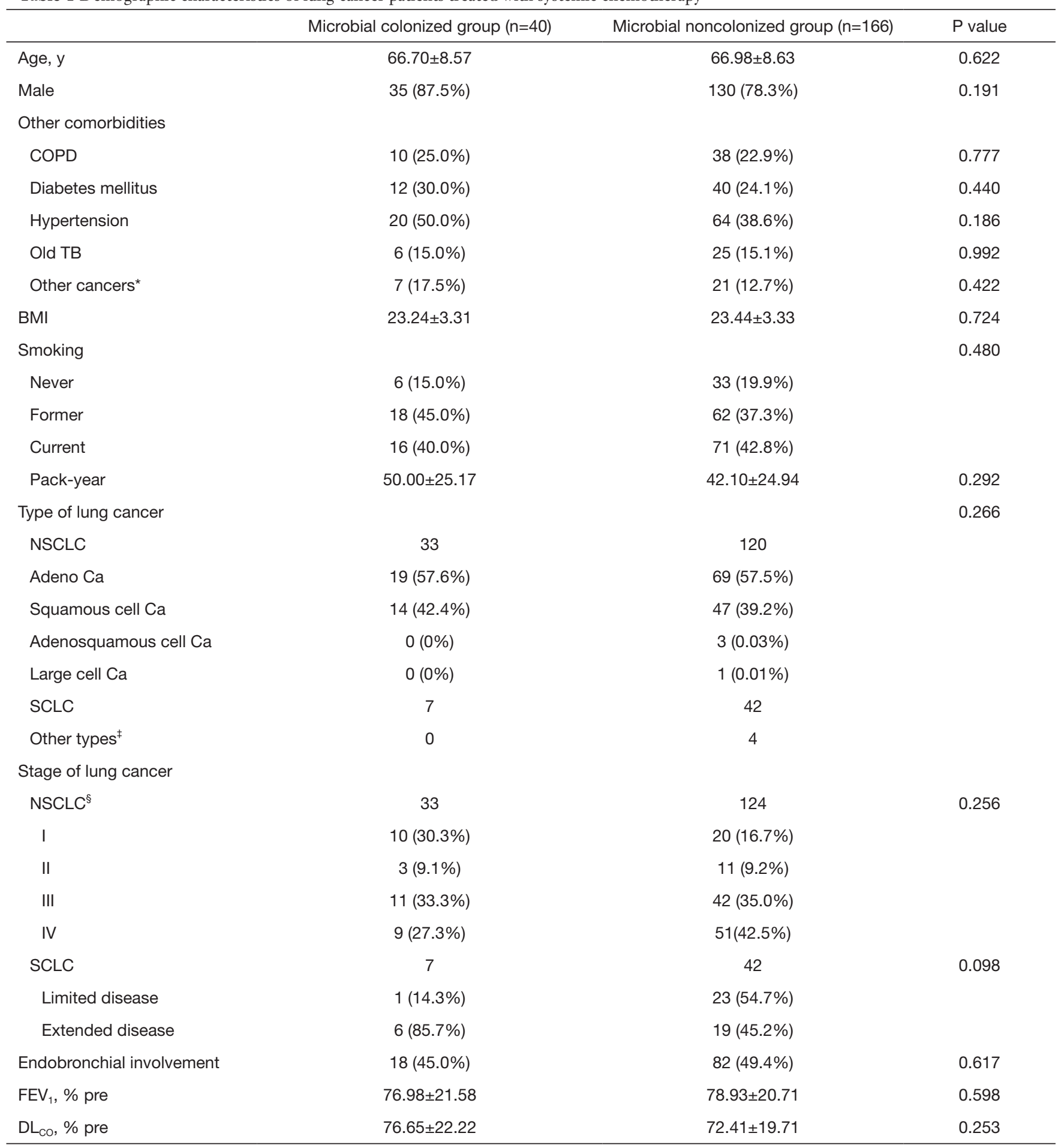

The data are presented as mean \pm SD or $\mathrm{n}(\%)$ unless otherwise indicated. ${ }^{*}$ Other cancers (n): stomach ca (8), hepatocellular ca (2), bladder ca (3), uterine ca (1), thyroid ca (1), ovary ca (1), glottic ca (1), pancreatic ca (1), tonsil ca (1), rectal ca (1); ${ }^{\ddagger}$ Other types (n): pleomorphic carcinoma (1), SCLC combined with adenocarcinoma component (1), SCLC combined with squamous cell carcinoma component (1), large cell neuroendocrine carcinoma (1); ${ }^{\circledR}$ NSCLC in this context included other types ${ }^{\ddagger}$ excluding SCLC. SD, standard deviation; COPD, chronic obstructive pulmonary disease; TB, tuberculosis; BMI, body mass index; NSCLC, non-small cell lung cancer; Ca, carcinoma SCLC, small cell lung cancer; $\mathrm{FEV}_{1}$, forced expiratory volume in one second; $\mathrm{DL}_{\mathrm{Co}}$, diffusion capacity of the lung for carbon monoxide. 
Table 2 Pattern of organisms isolated from initial bronchoscopy specimens in the microbial colonized group with lung cancer

\begin{tabular}{ll}
\hline Organism* $^{*}$ & $\mathrm{~N}$ \\
\hline Klebsiella pneumoniae & 14 \\
Streptococcus pneumoniae & 6 \\
Staphylococcus aureus & 5 \\
Pseudomonas aeruginosa & 2 \\
Raoultella planticola & 2 \\
Citrobacter freundii & 1 \\
Enterobacter aerogenes & 1 \\
Morganella morganii & 1 \\
Proteus mirabilis & 1 \\
Staphylococcus agalactiae & 1 \\
Mycobacterium tuberculosis & 1 \\
Nontuberculous mycobacterium & 4 \\
Aspergillus spp. & 3 \\
Candida glabrata & 2 \\
Mucormycosis & 1 \\
\hline
\end{tabular}

*More than one organism was cultured in some patients.
Table 4 Patterns of isolated organisms from respiratory specimens obtained from the 47 cases of pneumonia developed after chemotherapy for lung cancer

\begin{tabular}{lc}
\hline Organism* $^{*}$ & Number \\
\hline Acinetobacter baumannii & 3 \\
Klebsiella pneumoniae & 5 \\
Streptococcus pneumoniae & 2 \\
Staphylococcus aureus & 8 \\
Pseudomonas aeruginosa & 2 \\
Mycoplasma pneumoniae & 2 \\
Escherichia coli & 1 \\
Klebsiella oxytoca & 1 \\
Stenotrophomonas maltophilia & 1 \\
Rothia mucilaginosa & 1 \\
Mycobacterium tuberculosis & 1 \\
Nontuberculous mycobacterium & 6 \\
Pneumocystis jirovecii & 12 \\
Aspergillus spp. & 4 \\
Virus & (1 influenza, \\
& (Mainfluenza) \\
\hline
\end{tabular}

*More than one organism was detected in some patients.

Table 3 Pneumonia episodes and clinical outcomes according to bronchoscopy surveillance cultures in lung cancer patients treated with systemic chemotherapy

\begin{tabular}{|c|c|c|c|}
\hline & \multicolumn{2}{|c|}{ Bronchoscopy results } & $P$ value \\
\hline Interval from the initial bronchoscopy to the first pneumonia, days & $129(24-664)$ & $174(15-1,349)$ & 0.421 \\
\hline Admission due to pneumonia & $15(37.5 \%)$ & $66(39.9 \%)$ & 0.645 \\
\hline Neutropenia during chemotherapy & $15(37.5 \%)$ & $82(49.4 \%)$ & 0.176 \\
\hline Overall mortality & $22(55.0 \%)$ & $100(60.2 \%)$ & 0.545 \\
\hline
\end{tabular}

The data are presented as $\mathrm{n}(\%)$ unless otherwise indicated. 


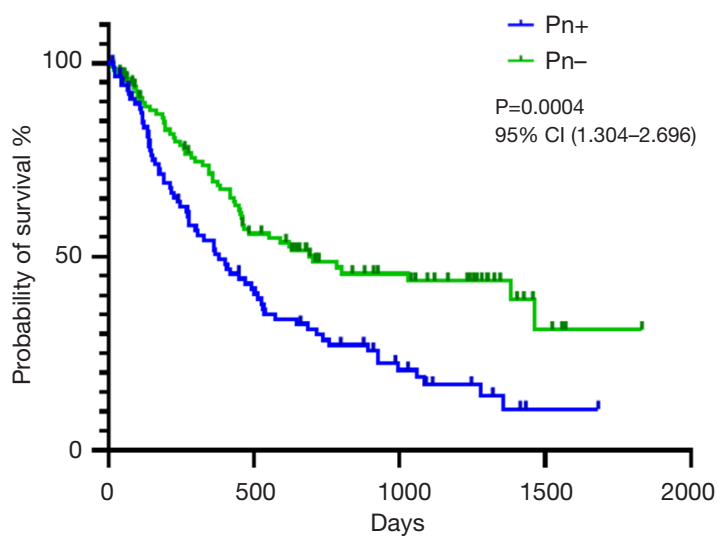

Figure 2 Comparison of survival probability according to occurrence of pneumonia in lung cancer patients treated with chemotherapy. Median overall survival 378 days in pneumonia group vs. 705 days in non-pneumonia group. Pn+, development of pneumonia; Pn-, no development of pneumonia; CI, confidence interval.

with our results $(11,17)$. This controversial result may reflect differences in the study population, use of various methods to acquire respiratory samples, and definition of colonization.

Our study produced some peculiar findings. There were no cases in which the initial cultured organisms in the microbial colonized group led to subsequent pneumonia after chemotherapy. In addition, the mean interval from the bronchoscopy evaluation of colonization to the detection of pneumonia was $>4$ months in both the colonized and non colonized groups, which is longer than the mean interval of $<1$ month in previous studies $(7,10,16)$, all of which reported an association between colonization and pneumonia. An interval of 4 months appears to be sufficient for colonized organisms to change or disappear. At the time of pneumonia after chemotherapy, P. jirovecii was the most commonly detected pathogen, and other opportunistic microbes such as NTM and virus were frequently isolated. By contrast, the two major bacteria species isolated in the initial surveillance culture, $K$. pneumoniae and $S$. pneumoniae, did not contribute significantly to later pneumonia development. These results lead us to suggest that surveillance culture of the airway, especially in patients without initial evidence of pneumonia, may not be a good predictor of the later development of pneumonia or mortality in lung cancer patients, at least in our setting.

We confirmed the previous finding that the occurrence of pneumonia caused poor prognosis in lung cancer patients who received chemotherapy irrespective of the presence of initial colonization (18). The incidence of pneumonia development among lung cancer patients in the current study was $43.2 \%$, higher than the $33 \%$ reported in the others $(11,18)$. The difference rates may be due to differences in the study populations. Onethird of the patients in our study had early lung cancer (stage I/II non-small cell lung cancer and limited disease in small cell lung cancer) and, consequently, they had a longer follow-up, which was reflected in a higher rate of diagnosing pneumonia development. Several possible reasons may explain why lung cancer is associated with frequent occurrence of pneumonia. Defects in the lungclearance system, such as low mucociliary function because of chemotherapy or structural deformity due to bronchial involvement in the tumor, are a crucial mechanism of pneumonia development $(19,20)$. Another important cause of respiratory infection, especially by opportunistic pathogens such as $P$. jirovecii or cytomegalovirus, is the presence of an immunosuppressive state, including an imbalance of T-lymphocyte subsets because of intensive chemotherapy or malnutrition (20). Interestingly, we observed that the microbial colonized group had a shorter time to pneumonia development than the noncolonized group, although the difference was not statistically significant. This finding suggests that some patients' characteristics that increase the risk for colonization may also make them vulnerable to the occurrence of pneumonia.

Recently, the incidence of nontuberculous mycobacterial lung disease has increased rapidly in South Korea as well as globally (21). In our study, four patients with lung cancer had a positive culture for NTM in the initial bronchoscopy specimens and were followed up without management for it. Only one had persistent culture for NTM ( $M$. intracelluare) and experienced slow progression of the lung lesion by the microbe. In addition, NTM was not previously isolated in the six patients whose samples were positive for this organism at the time of pneumonia development, and one case had result for subspecies identification ( $M$. massiliense). Interestingly, all of the above patients whose samples exhibited NTM were not treated, and only a few cases were tested to identify the subspecies. To confirm that NTM is a true pathogen or colonization, clinicians should monitor relevant findings in NTM lung disease and repeat microbiologic tests including verification at subspecies level.

Our study has some limitations. First, this was a retrospective study. The microbes tested in the initial 
surveillance did not include various opportunistic pathogens diagnosed using the PCR test, and these microbes were substantial causes of pneumonia development in our study. However, clinically, it is not possible to assess every organism only for surveillance considering the high costs involved and lack of medical resources. We believe that the results of our study reflect the situation in real practice. Second, 14 of 40 patients in the colonized group were treated with antibiotic therapy during the initial admission. A main reason for antibiotics use was that patients had respiratory symptoms such as dyspnea, hemoptysis or cough/ sputum, but not typical features of pneumonia, and the clinicians decided to prescribe the medication empirically. Although a type of antibiotics was not changed after obtaining the microbial results, the antibiotics use might be a crucial factor to clear the colonized bacteria. Third, we differentiated colonization from infection according to a clinical decision, but the quantification of microorganisms is a more accurate and objective way to define colonization. Finally, because clinical and radiographic findings may not always be apparent because of a disordered host inflammatory response in neutropenic cancer patients, it is possible that some pneumonia patients were not included.

In conclusion, we found that the rate of microbial colonization in initial bronchoscopy specimens was $19.4 \%$ in patients with lung cancer who underwent subsequent chemotherapy and bronchial colonization was not associated with pneumonia development or mortality after chemotherapy in these patients. These findings suggest that surveillance culture in the airway may not to be helpful to predict pneumonia or to improve survival in lung cancer patients, at least in a clinical setting similar to ours.

\section{Acknowledgments}

Funding: None.

\section{Footnote}

Reporting Checklist: The authors have completed the STROBE reporting checklist. Available at http://dx.doi. org/10.21037/jtd-20-2722

Data Sharing Statement: Available at http://dx.doi. org/10.21037/jtd-20-2722

Conflicts of Interest: All authors have completed the ICMJE uniform disclosure form (available at http://dx.doi. org/10.21037/jtd-20-2722). The authors have no conflicts of interest to declare.

Ethical Statement: The authors are accountable for all aspects of the work in ensuring that questions related to the accuracy or integrity of any part of the work are appropriately investigated and resolved. The study was conducted in accordance with the Declaration of Helsinki (as revised in 2013). This study was approved by the Clinical Research Ethics Committee of the Catholic Medical Center (XC140IMI0070). Due to retrospective nature of this study, the need for patient consents was waived.

Open Access Statement: This is an Open Access article distributed in accordance with the Creative Commons Attribution-NonCommercial-NoDerivs 4.0 International License (CC BY-NC-ND 4.0), which permits the noncommercial replication and distribution of the article with the strict proviso that no changes or edits are made and the original work is properly cited (including links to both the formal publication through the relevant DOI and the license). See: https://creativecommons.org/licenses/by-nc-nd/4.0/.

\section{References}

1. Evans SE, Ost DE. Pneumonia in the neutropenic cancer patient. Curr Opin Pulm Med 2015;21:260-71.

2. Roychowdhury M, Pambuccian SE, Aslan DL, et al. Pulmonary complications after bone marrow transplantation: an autopsy study from a large transplantation center. Arch Pathol Lab Med 2005;129:366-71.

3. Lanoix JP, Pluquet E, Lescure FX, et al. Bacterial infection profiles in lung cancer patients with febrile neutropenia. BMC Infect Dis 2011;11:183.

4. Lee JO, Kim DY, Lim JH, et al. Risk factors for bacterial pneumonia after cytotoxic chemotherapy in advanced lung cancer patients. Lung cancer 2008;62:381-4.

5. Gabazza E, Taguchi O, Yamakami T, et al. Obstructive pneumonitis in lung cancer patients--a retrospective study. Nihon Kyobu Shikkan Gakkai Zasshi 1992;30:1820-4.

6. Kim YJ, Kim SI, Jun YH, et al. Clinical significance of surveillance culture in liver transplant recipients. Transplant Proc 2014;46:828-31.

7. Kabak E, Hudcova J, Magyarics Z, et al. The utility of endotracheal aspirate bacteriology in identifying mechanically ventilated patients at risk for ventilator associated pneumonia: a single-center prospective 
observational study. BMC Infect Dis 2019;19:756.

8. Dray S, Coiffard B, Persico N, et al. Are tracheal surveillance cultures useful in the intensive care unit? Ann Transl Med 2018;6:421.

9. Gao K, Lai Y, Huang J, et al. Preoperatiove Airway Bacterial Colonization: the Missing Link between Nonsmall Cell Lung Cancer Following Lobectomy and Postoperative Pneumonia? Zhongguo Fei Ai Za Zhi 2017;20:239-47.

10. Belda J, Cavalcanti M, Ferrer M, et al. Bronchial colonization and postoperative respiratory infections in patients undergoing lung cancer surgery. Chest 2005;128:1571-9.

11. Dancewicz M, Szymankiewicz M, Bella M, et al. Bronchial bacterial colonization in patients with lung cancer.

Pneumonol Alergol Pol 2009;77:242-7.

12. Laroumagne S, Lepage B, Hermant C, et al. Bronchial colonisation in patients with lung cancer: a prospective study. Eur Respir J 2013;42:220-9.

13. Clinical and Laboratory Starndards Institute. Performance Standards for Antimicrobial Susceptibility Testing: Twentieth Informational Supplement. Wayne, PA: Clinical and Laboratory Standards Institute, 2012.

14. Zirakzadeh A, Patel R. Vancomycin-resistant enterococci: colonization, infection, detection, and treatment. Mayo

Cite this article as: Kang JY, Kang HS, Heo JW, Kim YH, Kim SJ, Lee SH, Kwon SS, Kim YJ. Clinical significance of microbial colonization identified by initial bronchoscopy in patients with lung cancer requiring chemotherapy. J Thorac Dis 2021;13(3):1306-1314. doi: 10.21037/jtd-20-2722
Clin Proc 2006;81:529-36.

15. Kim YJ, Kim SI, Kim YR, et al. Risk factors for vancomyin-resistant enterococci infection and mortality in colonized patients on intensive care unit admission. Am J Infect Control 2012;40:1018-9.

16. Schussler O, Alifano M, Dermine H, et al. Postoperative pneumonia after major lung resection. Am J Respir Crit Care Med 2006;173:1161-9.

17. Yosuke Togashi, Katsuhiro Masago, Yutaka Ito, et al. Pneumocystis jiroveci pneumonia and colonization in patients with advanced lung cancer. Oncol Lett. 2013;5:601-4.

18. Perlin E, Bang KM, Shah A, et al. The impact of pulmonary infections on the survival of lung cancer patients. Cancer 1990;66:593-6.

19. Sayit E, Aktogu S, Ertay T, et al. Effect of chemotherapy on pulmonary epithelial permeability in lung cancer. Nucl Med Biol 2000;27:581-5.

20. Oizumi K. Respiratory infectious complications in patients with lung cancer. Nihon Kyobu Shikkan Gakkai Zasshi 1989;27:286-8.

21. Park SC, Kang MJ, Han CH, et al. Prevalence, incidence, and mortality of nontuberculous mycobacterial infection in Korea:a nationwide population-based study. BMC Pulm Med 2019.19;140. 